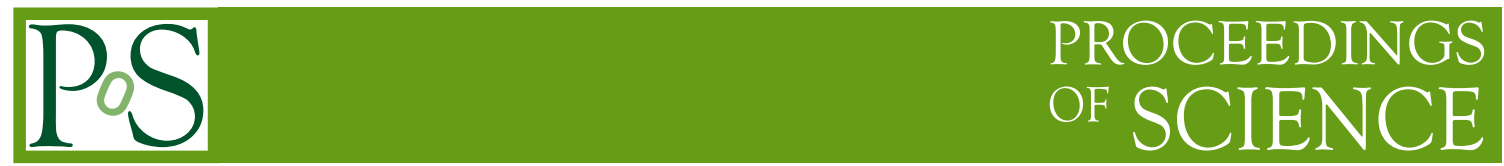

\title{
Searches for Dark Photons at LHCb
}

\section{Cid Vidal ${ }^{a \dagger *}$}

${ }^{a}$ Instituto Galego de Física de Altas Enerxías, Universidade de Santiago de Compostela, Rúa de Xoaquín Díaz de Rábago, s/n, 15782 Santiago de Compostela, Galicia, Spain

E-mail: xabier.cid.vidal@cern.ch

In the past years, LHCb has shown its capabilities to perform interesting searches for beyond the Standard Model particles. The most clear example of this is that of Dark Photons, which are hypothetical new particles that serve as a "portal" between the Standard Model and a potential hidden dark sector. These proceedings explain the searches for Dark Photons decaying to a $\mu \mu$ pair that LHCb has performed with its 2016-2018 dataset, as well as a model independent search to a new particle that would also decay to a $\mu \mu$ pair, covering several different interesting topologies.

40th International Conference on High Energy physics - ICHEP2020

July 28 - August 6, 2020

Prague, Czech Republic (virtual meeting)

\footnotetext{
$\dagger$ On behalf of the LHCb collaboration

* Speaker
} 


\section{Introduction}

These proceedings discuss the searches for dark photons that the LHCb experiment at CERN has performed in the last years. Dark photons (DPs) [1] appear in some of the most promising Standard Model (SM) extensions. They are hypothetical particles that play a role similar to SM photons in hidden dark sectors. DPs couple via kinetic mixing to the SM photons, providing a "portal" that connects these sectors to the SM. Note the magnitude of this coupling affects both the production rate and lifetime of DPs. Searches for dark photons decaying to muons at LHCb are presented in this document together with a new model independent search where the final state $\mu \mu$ is targeted in a more inclusive way, covering more topologies than for DPs.

$\mathrm{LHCb}[2,3]$ is a forward spectrometer with special features that make it specially suited for searches for low-mass $\mu \mu$ resonances. Some of these features are an advanced vertexing or an excellent momentum resolution and particle identification. This is complemented by a trigger that is capable of selecting very soft objects when compared to other experiments at the LHC [4]. This will be reinforced at the next run of the LHC, beyond 2021, when LHCb will be upgraded to be readout at every bunch-crossing, allowing a trigger fully-based on software, which is very convenient to detect the type of signatures presented in this document.

\section{Searches for dark photons}

DPs can be detected through different production modes and different decay topologies. Several proposals exist to search for DPs at LHCb $[5,6]$. The first covers the production of DPs in $D^{*}$ decays, with DPs decaying to an $e e$ pair, while the second the inclusive production of DPs which later decay to a $\mu \mu$ pair. The DP decay can be displaced from the primary vertex (PV) or occurring promptly.

Two different searches are performed at LHCb using the second of the strategies above, including prompt and displaced DP decays to $\mu \mu$. The datasets used in these searches were recorded in the 2016-2018 period [7, 8]. Note that, when decaying to $\mu \mu$, the production of DPs self-normalizes to the SM $\gamma^{*} \rightarrow \mu \mu$ process so that, in general, no absolute efficiencies need to be computed. Figure 1 shows the prompt $\mu \mu$ spectra detected at LHCb with the 2016 dataset. The yields of the different SM resonances, such as $\eta \rightarrow \mu \mu, \phi \rightarrow \mu \mu$ or $J / \psi \rightarrow \mu \mu$ indicate the sensitivity of LHCb to detect a DP. Although no DP is found by these searches, the limits achieved are world-best in different regions of the parameter space of the "minimal" DP [1]. This is particularly true in the region below $1 \mathrm{GeV} / \mathrm{c}^{2}$. Figure 2 shows the parameter space regions excluded by the analyses presented in this document.

Note that the future datasets [9] to be recorded by LHCb will increase the sensitivity of the experiment in the search for DPs. This will come from the upgrade of the trigger, described above, and for the increase in the size of these datasets. The sensitivity that $\mathrm{LHCb}$ and other experiments will achieve in the future is described in detail in Ref. [10].

\section{Model independent $\mu \mu$ searches}

Other than DPs, the $\mu \mu$ final state appears in several beyond the SM (BSM) models as a promising way to detect new particles. Following this, a general, model independent, search is 


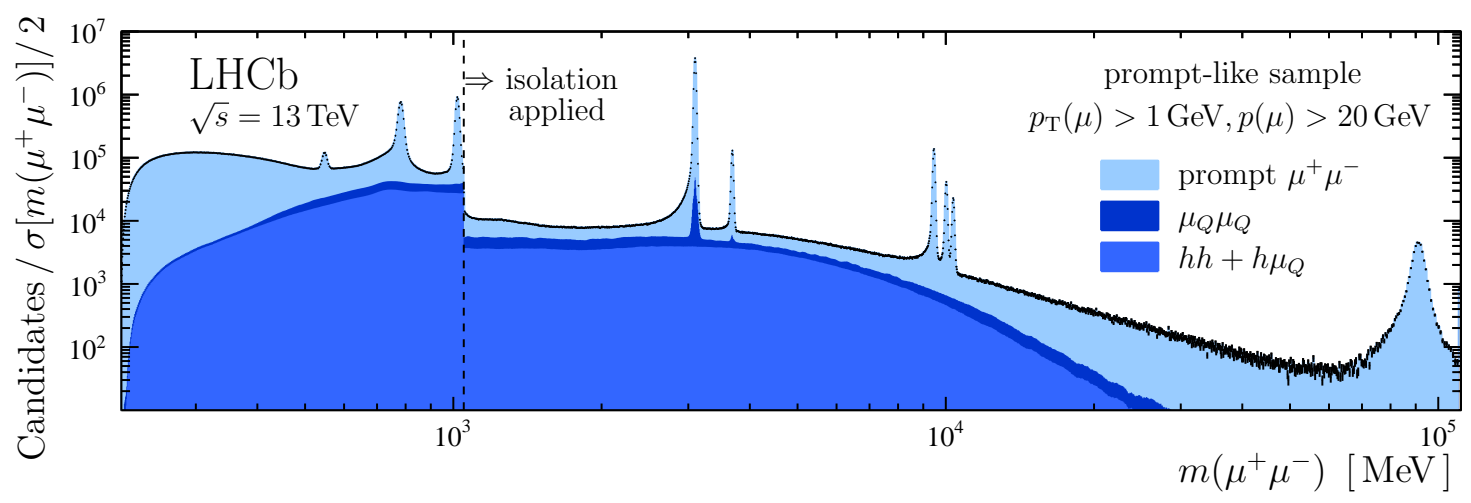

Figure 1: The $\mu^{+} \mu^{-}$prompt spectra recorded with the $2016 \mathrm{LHCb}$ dataset. The darker blue components indicate the backgrounds originating from heavy QCD decays $\left(\mu_{Q} \mu_{Q}\right)$ or hadrons misidentified as muons $\left(h h+h \mu_{Q}\right)$. Figure taken from Ref. [7].

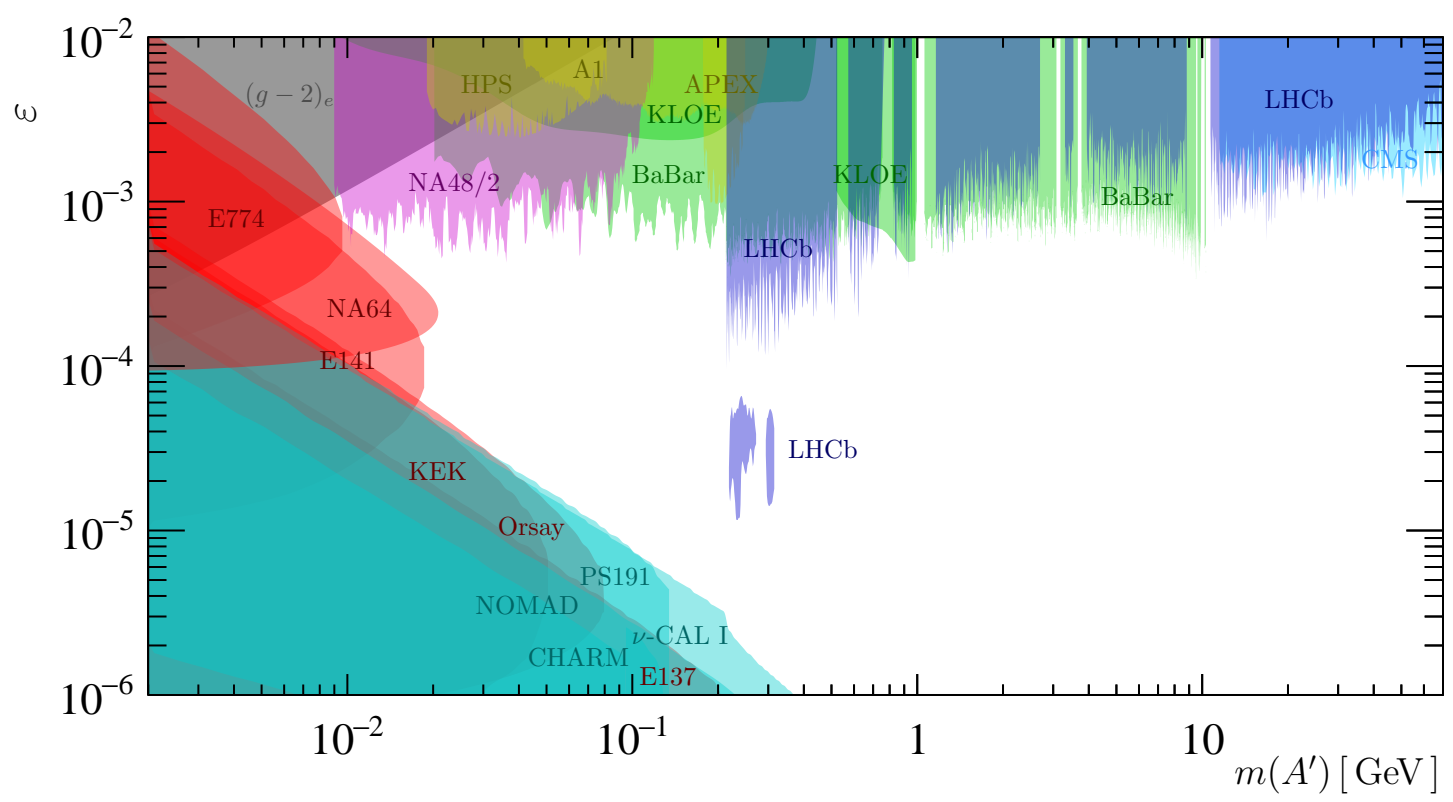

Figure 2: The minimal dark photon parameter space currently excluded by different experiments. The $y$ axis represents the kinematic mixing strength between the dark and SM photon, while the $x$ axis the mass of the dark photon. Figure taken from Ref. [8], where the full list of experimental results covered in this plot can be found.

also performed at LHCb [11] using the same dataset as the DP searches, but with slightly different selection requirements. These relaxed requirements increase the sensitivity to search for these new resonances $(X \rightarrow \mu \mu)$ appearing in different types of BSM models. For this analysis, efficiencies are obtained from simulation as a function of different parameters of the $X$ resonance, hence allowing to obtain model-independent results as a function of these parameters. Examples are the $X$ mass, $p_{T}$ or natural width. To cover as many models as possible, this search includes both prompt or displaced decays of the $X$ resonance, and also the possibility that these might not point back to the $\mathrm{PV}$, to cover the production of the $X$ in decays of other displaced new particles. Finally, the 


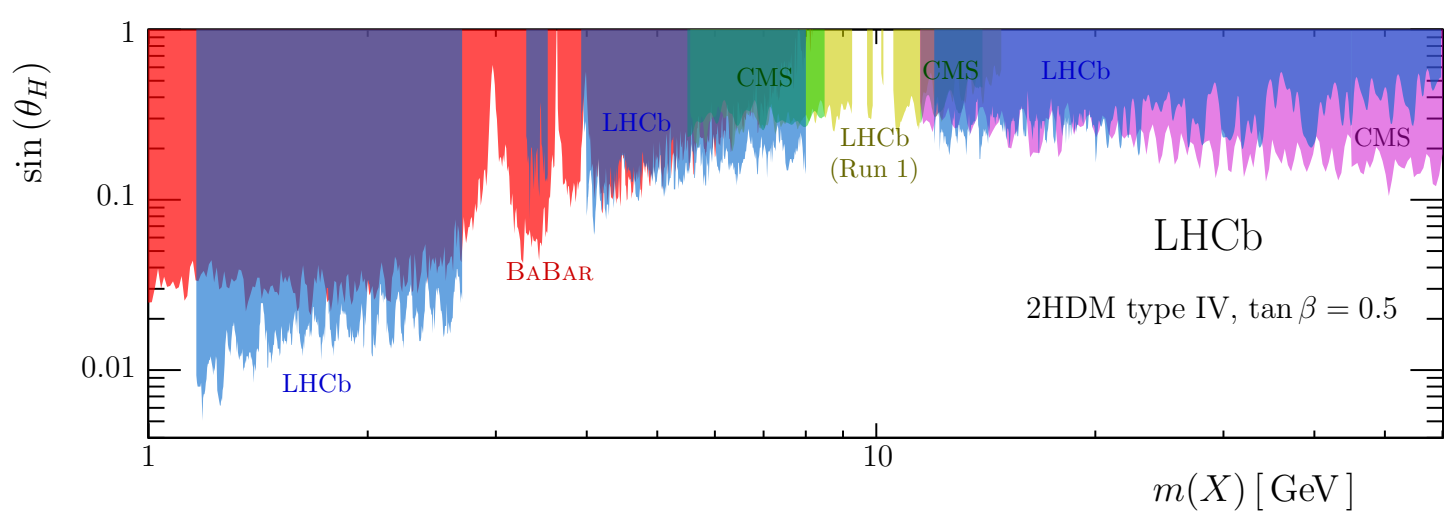

Figure 3: The pseudoscalar $(X)$ - Higgs mixing angle versus the $X$ mass space for a realization of the 2HDM model [12]. Figure taken from Ref. [11]. The experimental exclusions include results from LHCb [11, 14], CMS [15, 16], and BaBar [17].

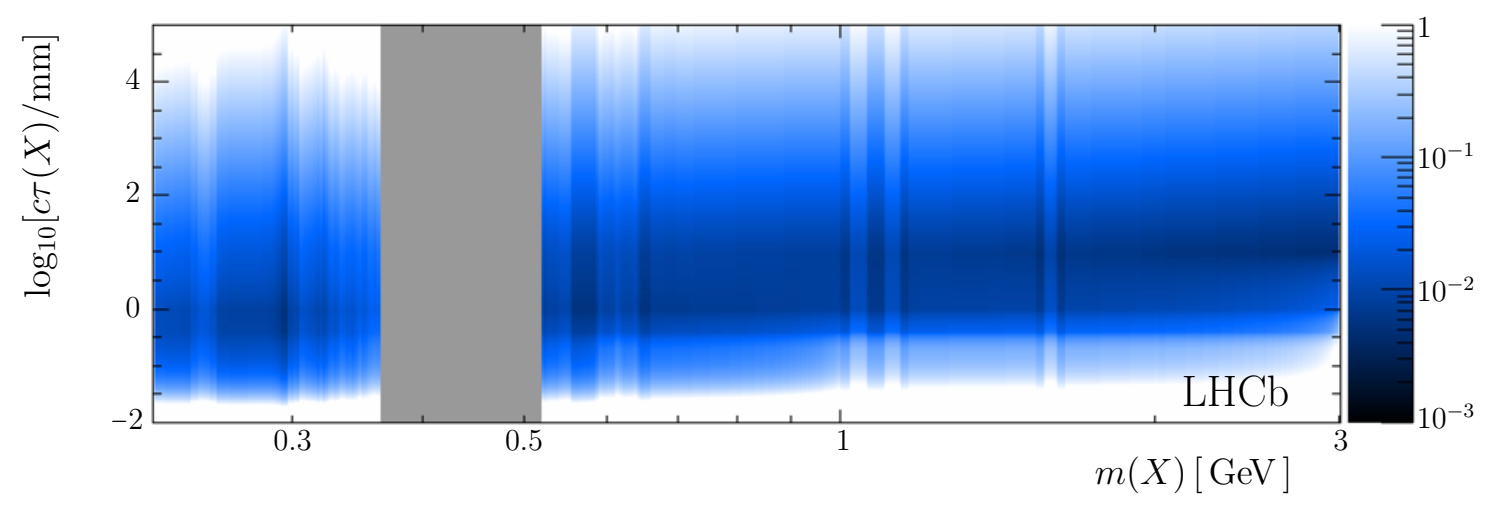

Figure 4: Upper limits in the $Z_{\mathrm{HV}}-\gamma$ kinetic mixing strength in the HV scenario [13] for different masses and lifetimes of the hidden hadron. Figure taken from Ref. [11].

production of the $X$ resonance in association to $b$-jets is considered too. As for dark photons, no excess is found, but the results achieved are world-best in several regions of the parameter space of the $X$ particles.

Apart from the model independent results, interpretations are provided in a couple of theoretical models of interest. For the first, which involves a realization of the 2HDM scenario [12], the regions excluded by this result are showed in Figure 3. In this case the expected signal is a simple prompt $\mu \mu$ light resonance. For the second, the phenomenology involves displaced decays of a hidden hadron in Hidden Valley (HV) models [13]. This result allows setting constraints on the kinetic-mixing strength between the photon and a heavy HV boson, $Z_{\mathrm{HV}}$. Note, that, since the kinematics of the hidden hadrons depend on the average HV hadron multiplicity, this number is fixed to around 10 for all hidden hadron masses. The results are presented in Figure 4. 


\section{Conclusions}

These proceedings show that $\mathrm{LHCb}$ is at the moment much more than a flavor factory. The $\mu \mu$ topology offers an excellent example of this, providing world-best sensitivity to very popular BSM models, such as that of DPs. LHCb could become our best tool to provide the discovery of a BSM particle in the future, similar to other proposed experiments [18], but having the big advantage that it is already built and running.

\section{References}

[1] M. Fabbrichesi, E. Gabrielli, and G. Lanfranchi, The Dark Photon, arXiv:2005. 01515.

[2] LHCb collaboration, A. Alves et al., The LHCb Detector at the LHC, JINST 3 (2008) S08005.

[3] LHCb collaboration, R. Aaij et al., LHCb Detector Performance, Int. J. Mod. Phys. A30 (2015) 1530022, arXiv: 1412.6352.

[4] LHCb collaboration, LHCb Trigger and Online Upgrade Technical Design Report, CERNLHCC-2014-016. LHCB-TDR-016, 2014.

[5] P. Ilten, J. Thaler, M. Williams, and W. Xue, Dark photons from charm mesons at LHCb, Phys. Rev. D 92 (2015) 115017, arXiv: 1509. 06765.

[6] P. Ilten et al., Proposed Inclusive Dark Photon Search at LHCb, Phys. Rev. Lett. 116 (2016) 251803, arXiv: 1603.08926.

[7] LHCb collaboration, R. Aaij et al., Search for dark photons produced in $13 \mathrm{TeV}$ pp collisions, Phys. Rev. Lett. 120 (2018) 061801, arXiv: 1710.02867.

[8] LHCb collaboration, R. Aaij et al., Search for $A^{\prime} \rightarrow \mu^{+} \mu^{-}$Decays, Phys. Rev. Lett. 124 (2020) 041801, arXiv: 1910.06926.

[9] LHCb collaboration, Expression of Interest for a Phase-II LHCb Upgrade: Opportunities in flavour physics, and beyond, in the HL-LHC era, CERN-LHCC-2017-003, CERN, Geneva, 2017.

[10] X. Cid Vidal et al., Report from Working Group 3: Beyond the Standard Model physics at the HL-LHC and HE-LHC, CERN Yellow Rep. Monogr. 7 (2019) 585, arXiv: 1812.07831.

[11] LHCb collaboration, R. Aaij et al., Searches for low-mass dimuon resonances, arXiv:2007.03923.

[12] U. Haisch and J. F. Kamenik, Searching for new spin-0 resonances at LHCb, Phys. Rev. D 93 (2016) 055047, arXiv: 1601.05110.

[13] A. Pierce, B. Shakya, Y. Tsai, and Y. Zhao, Searching for confining hidden valleys at LHCb, ATLAS, and CMS, Phys. Rev. D 97 (2018) 095033, arXiv: 1708.05389. 
[14] LHCb collaboration, R. Aaij et al., Search for a dimuon resonance in the $\Upsilon$ mass region, JHEP 09 (2018) 147, arXiv: 1805.09820.

[15] CMS collaboration, S. Chatrchyan et al., Search for a Light Pseudoscalar Higgs Boson in the Dimuon Decay Channel in pp Collisions at $\sqrt{s}=7$ TeV, Phys. Rev. Lett. 109 (2012) 121801, arXiv: 1206.6326.

[16] CMS collaboration, A. M. Sirunyan et al., Search for a Narrow Resonance Lighter than 200 $\mathrm{GeV}$ Decaying to a Pair of Muons in Proton-Proton Collisions at $\sqrt{s}=\mathrm{TeV}$, Phys. Rev. Lett. 124 (2020) 131802, arXiv: 1912 . 04776.

[17] BaBar collaboration, J. P. Lees et al., Search for di-muon decays of a low-mass Higgs boson in radiative decays of the Y(1S), Phys. Rev. D 87 (2013) 031102, arXiv: 1210. 0287, [Erratum: Phys.Rev.D 87, 059903 (2013)].

[18] J. Beacham et al., Physics Beyond Colliders at CERN: Beyond the Standard Model Working Group Report, J. Phys. G 47 (2020) 010501, arXiv: 1901. 09966. 